\title{
Macronutrient composition has sex specific effects on the GH-IGF-I axis
}

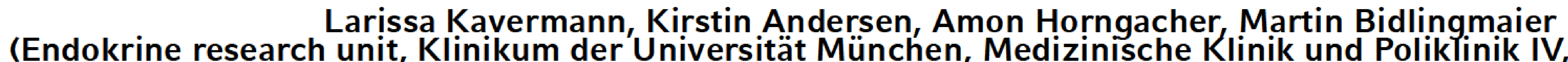

Contact: Martin.Bidlingmaier@med.Imu.de / KAnderse@med.Imu.de

\section{Introduction}

Metabolic diseases are among the most important diseases in western societies and usually related to obesity. Therefore typically dietary interventions are recommended for treatment and include both strategies with reduced caloric intake and alterations in macronutrient composition. Assuming that not only caloric density influences metabolism but also the specific makronutrient source itself. Furthermore metabolism must be assumed to be regulated differntly in females and males considering such facts as a natural given difference in body composition. In previous studies it has been established that low carbohydrate high fat (LCHF) diets affect the GH-IGF-I axis in male rats, we now investigated wether the same effects can be seen in females. We also included a high carbohydrate high fat (HCHF) diet in our study.

Methods

Purified diets (\% of metabolizable energy, fat/protein/carbohydrate: Chow (CH (16.7/19.0/64.3), protein matched LCHF-1 (78.7/19.1/2.2), ketogenic LCHF-2 (92.8/5.5/1.7), HCHF (61.9/18.7/19.4)) were pair-fed isoenergetically for 4 weeks to male and female Wistar rats (12 weeks at start, $n=7 /$ group). GH secretion profiles were collected (10 samples/5 hours) after 3 weeks. At study end (6 hours fasting, dark phase), blood samples and organs were collected. Pituitary GHRH-R and $\mathrm{GH}$ as well as liver GH-R and IGF-I mRNA were analyzed.

\section{Results}

Serum GH secretion showed higher nadirs and lower peaks in females. On the ketogenic LCHF-2 diet, GH tended to be lower in both sexes. Interestingly GH was increased only on HCHF in females. IGF-I $(\mathrm{ng} / \mathrm{mL})$ was significantly reduced on all HF diets in males (CH: 1441 \pm 79.13 , LCHF-1: 961.9*** \pm 31.48, LCHF-2: 940.2*** \pm 39.87 , HCHF: $993.6^{* * *} \pm 14.78$ ), but only on the ketogenic LCHF-2 diet in females (CH: 713.3 \pm 49.0 , LCHF-1: $588.0 \pm 48.15$, LCHF-2: $463.3^{* * *} \pm 34.58$, HCHF: 574.4 \pm 26.4 ) Pituitary GH mRNA was generally higher in females and lowest in rats of both sexes fed LCHF-2 diet. GHRH-R mRNA was reduced on all HF diets (males: CH: 0.07 \pm 0.01, LCHF-1: 0.04 \pm 0.01 , LCHF-2: 0.04 $\pm 0.01, \mathrm{HCHF}:$ $0.03 \pm 0.01 *$; females: CH: 0.08 \pm 0.01 , LCHF-1: 0.03 \pm 0.02, LCHF-2: $0.03 \pm 0.01$ * HCHF: $0.04 \pm 0.01$ ). Liver GH-R mRNA tended to be lower on LCHF-1 and LCHF-2 in both sexes and increased on HCHF in females.

\section{Conclusion and Discussion}

Our data demonstrate that HF diets lead to more pronounced deterioration of the GH-IGF-I axis in males. Still there can also be observed alterations in GH-IGF-I axis in female animals. Whether hypothalamic or pituitary mechanisms regulating $\mathrm{GH}$ secretion or peripheral mechanisms regulating $\mathrm{GH}$ sensitivity cause the sex differences is currently studied.

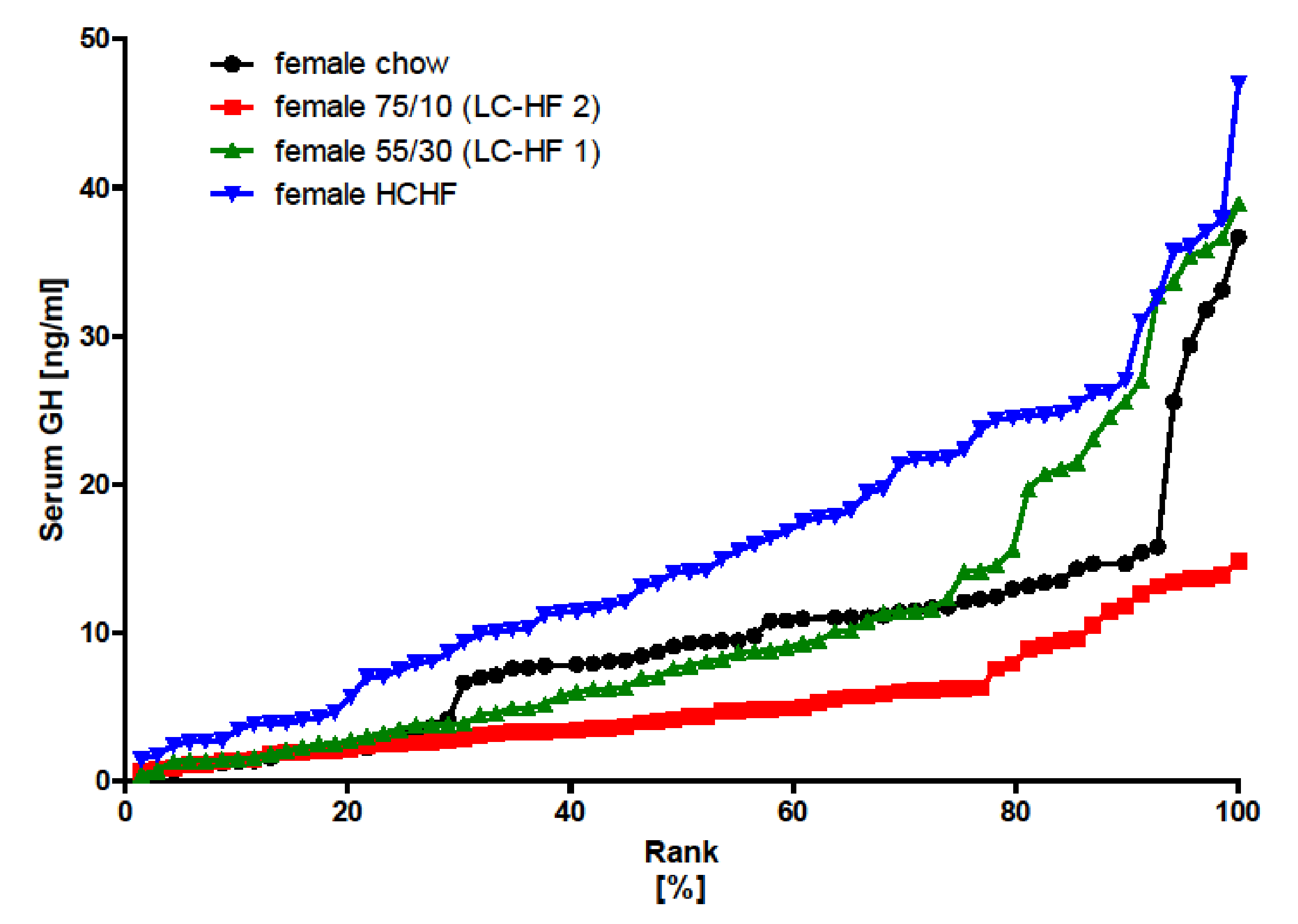

Fig.1: Serum GH levels which are decreased in all LCHF diets but increased on the HCHF diet in female rats

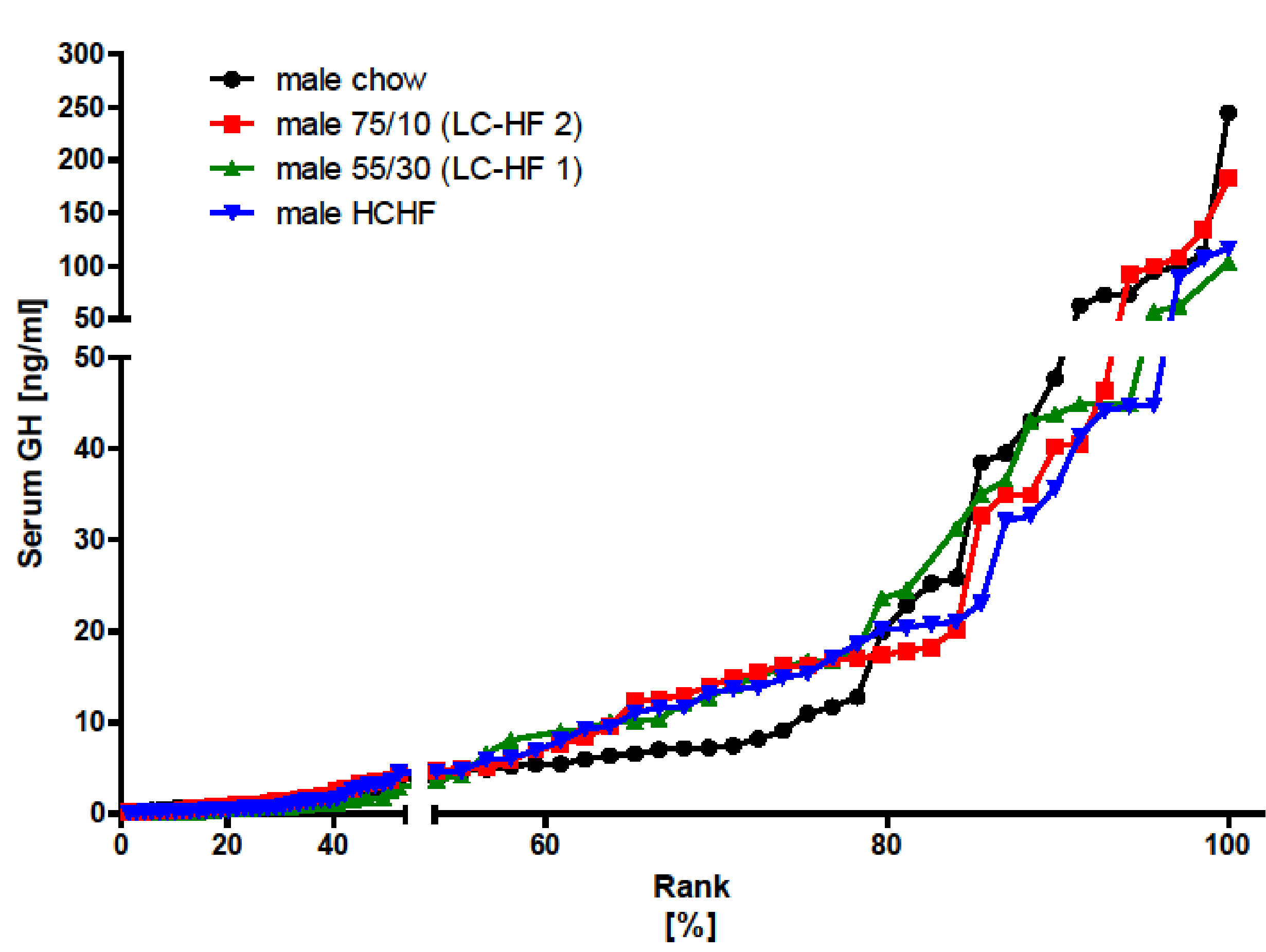

Fig.2: Serum GH levels which are decreased in all $\mathrm{HF}$ diets in males. In contrast to females the $\mathrm{GH}$ increase on a HCHF diet was not detectable in male rats

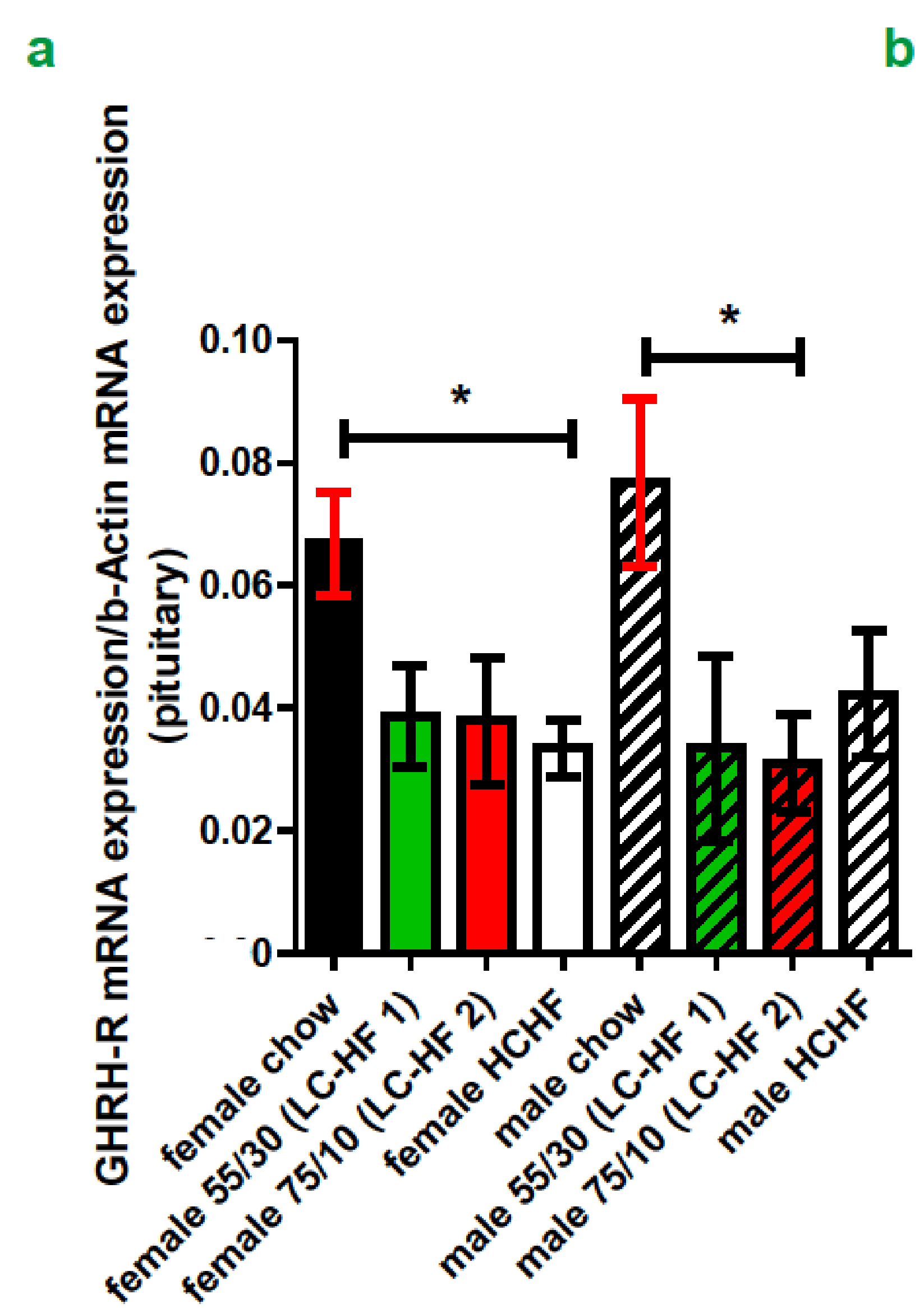

b

Fig.4: (a) Pituitary mRNA expression of GHRH-R is reduced on all HF diets in both sexes, (b) pituitary mRNA expressionof GH is is lowest on LCHF-2 in both sexes, however males did not show an increase in LCHF-1 as females did, (c) liver mRNA expression of GH-R tended to be lower on LC-diets in both sexes
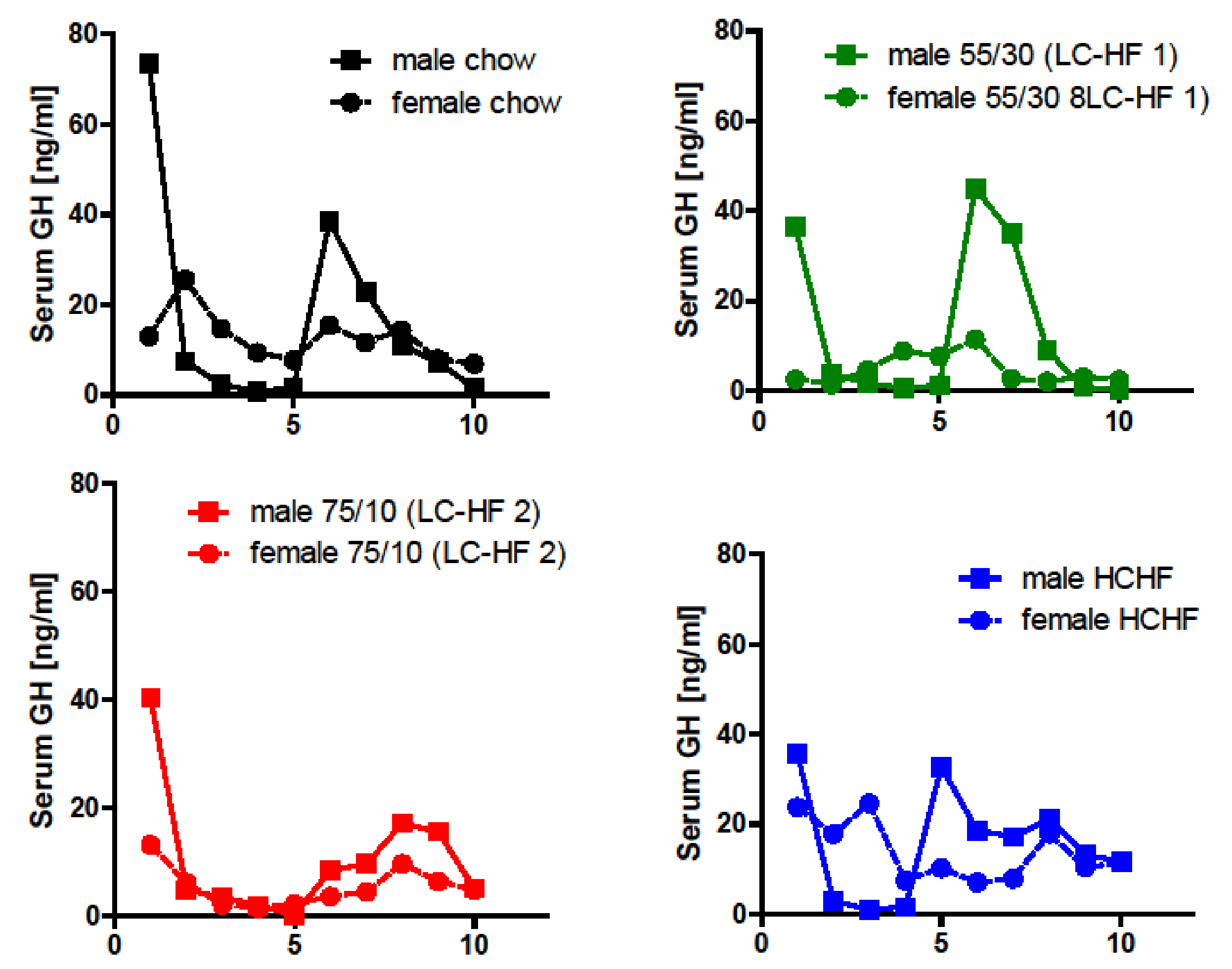

Fig.3: Females showedh igher nadirs and lower peaks in $\mathrm{GH}$ secreation compared to males. Both sexex had lower GH levels on the ketogenic LCHF-2 diets

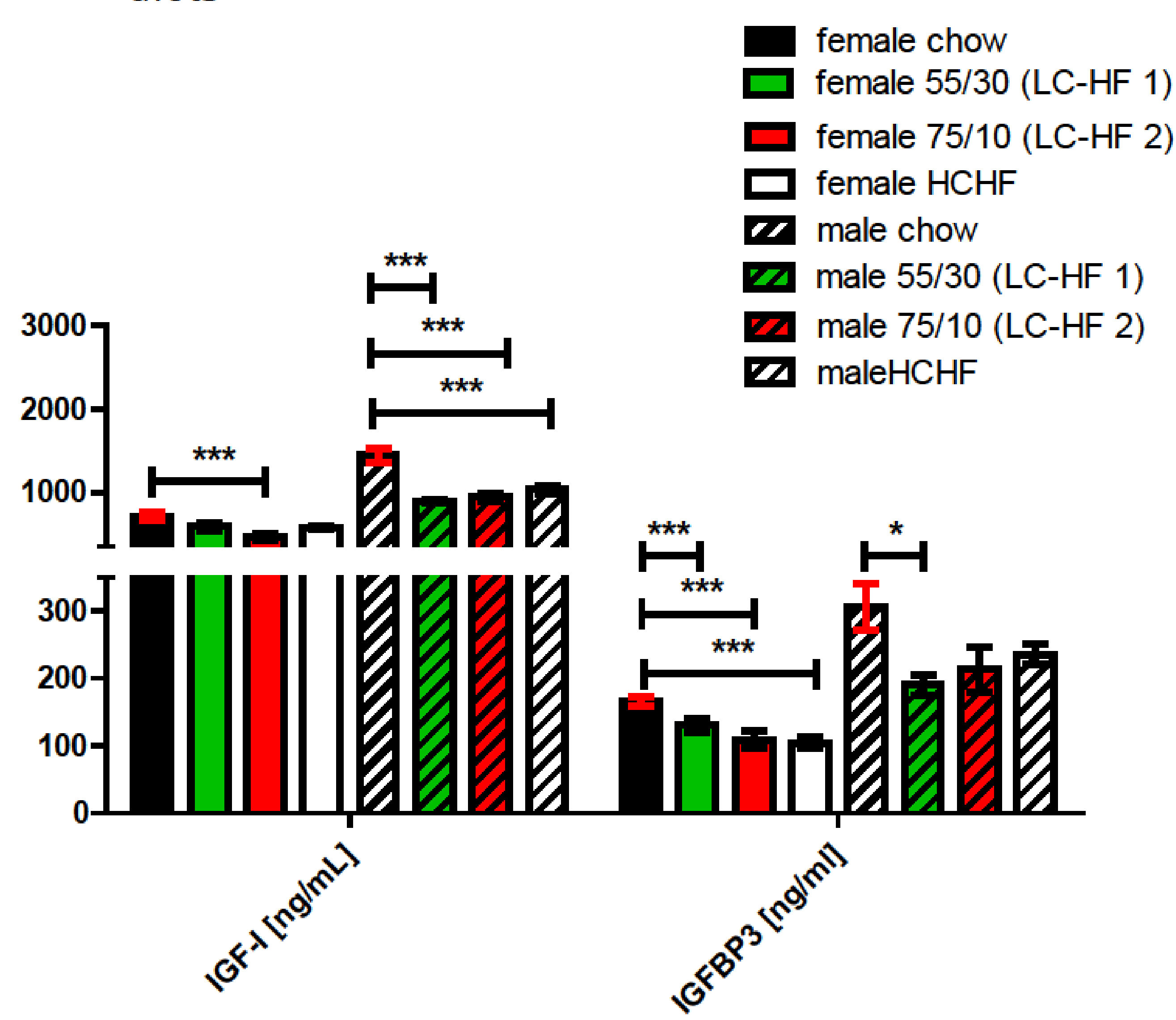

Fig.5: Serum IGF-I which is more significantly altered in male rats. Serum IGF-I-BP-3 which is more significantly reduced in female rats 